\title{
Gérard Bouchard, Raison et déraison du mythe. Au cour des imaginaires collectifs
}

Jean-François Plamondon

\section{(2) OpenEdition}

10 Journals

\section{Édition électronique}

URL : http://journals.openedition.org/studifrancesi/5467

DOI : 10.4000/studifrancesi.5467

ISSN : 2421-5856

Éditeur

Rosenberg \& Sellier

\section{Édition imprimée}

Date de publication : 1 décembre 2016

Pagination : 580-581

ISSN : 0039-2944

\section{Référence électronique}

Jean-François Plamondon, « Gérard Bouchard, Raison et déraison du mythe. Au cœur des imaginaires collectifs », Studi Francesi [En ligne], 180 (LX | III) | 2016, mis en ligne le 01 janvier 2017, consulté le 18 septembre 2020. URL : http://journals.openedition.org/studifrancesi/5467 ; DOI : https://doi.org/ 10.4000/studifrancesi.5467

Ce document a été généré automatiquement le 18 septembre 2020.

\section{(c)}

Studi Francesi è distribuita con Licenza Creative Commons Attribuzione - Non commerciale - Non opere derivate 4.0 Internazionale. 


\title{
Gérard Bouchard, Raison et déraison du mythe. Au cour des imaginaires collectifs
}

\author{
Jean-François Plamondon
}

\section{RÉFÉRENCE}

GÉRARD BOUCHARD, Raison et déraison du mythe. Au cœur des imaginaires collectifs, Montréal, Boréal, 2014, 230 pp.

1 La parution d'un livre de Gérard Bouchard au Québec est un événement. L'auteur est en effet considéré comme un des plus influents intellectuels contemporains. Sociologue et historien, il a notamment observé et analysé le comportement humain des petites communautés avant de prolonger sa réflexion sur l'ensemble québécois et de déborder sur l'ensemble du nouveau monde. En 2007 et 2008, il coprésidait avec le philosophe de réputation internationale, Charles Taylor, la commission sur les pratiques d'accommodement reliées aux pratiques culturelles dont le rapport devait servir de base aux futures politiques interculturelles du Gouvernement du Québec. C'est à ce titre d'ailleurs qu'il avait été invité à Bologne en 2009 lors d'un colloque organisé par le Centro Interuniversitario di Studi Quebecchesi qui portait sur la laïcité et la religion à l'heure des sociétés plurielles. Après avoir abordé les mythes dans un essai en 2003, il revient une dizaine d'années plus tard sur ce sujet pour tenter de construire une méthode d'analyse des mythes sociaux, puisque selon l'auteur aucune société ne peut se vanter d'être exempte de mythes. Malgré le progrès de la raison amenée par et depuis le siècle des Lumières, la raison n'explique pas tout ce qui meut les sociétés. L'émotion contrôle encore en grande partie les décisions apparemment raisonnées qui gouvernent les sociétés d'individus, pour paraphraser Elias. Pour Bouchard, l'émotion cimente en effet davantage que la raison puisqu'il s'agit d'un puissant mécanisme discursif «qui a le pouvoir de projeter toute une société dans les directions les plus diverses allant de l'altruisme le plus pur au fanatisme le plus destructeur» (p. 38). Le 
mythe, «comme représentation collective et comme mode particulier d'imputation de sens» (p.38), serait composé de six traits distinctifs: il est d'abord de fondement archétypal, il évolue et s'adapte ce qui lui donne un caractère hybride, l'émotion préside à son actualité, son discours est reconnu par ceux qui le portent comme inattaquable et jouit en ce sens d'une immunité du même type que celui du discours sacré, ses prêtres savent le narrer de façon à l'instrumentaliser à leurs fins et enfin le mythe se construit autour d'une histoire significative provenant du passé. À partir de ces quelques traits distinctifs, Bouchard propose ainsi une définition du mythe: «représentation collective hybride bénéfique ou nuisible, baignant dans le sacré, commandée par l'émotion plus que par la raison, et porteuse de sens, de valeurs et d'idéaux façonnés dans un environnement social et historique donné» (p. 41). Sans être une grande révolution, cette définition et la façon dont Bouchard mène sa réflexion placent le mythe à l'avant-plan d'une nouvelle pratique historiciste que l'on peut imaginer fructueuse. En effet, faire ressortir le mythe parmi les discours significatifs d'une société donnée, à une époque donnée, impose d'envisager une nouvelle histoire qui tiendra compte de ces mythes comme moteur d'une génération. Comme si toute raison était en fait encadrée et produite par un système discursif qui appartient à l'émotif. La raison trouve ainsi son fondement dans l'expérience émotive du moi et non dans sa part réfléchie et consciente. "En d'autres mots, ce sont les mythes, bien plus que les idées ou les idéologies, qui 'mènent le monde'. Ceci invite à rechercher derrière toute idée influente le mythe qui la soutient. On pourrait ainsi paraphraser La Rochefoucauld en affirmant que, dans l'idéologie, le recours au mythe est l'hommage que la raison rend à son contraire» (p.56). Intéressante perspective celle de vouloir expliquer par les passions et le cœur les tangentes empruntées par les peuples. Il est vrai que lorsque l'on enseigne l'histoire, on tente de raisonner parfois l'irraisonnable. Dans un numéro déjà paru de «Studi francesi», nous avons critiqué un essai de Marc Angenot intitulé L'Histoire des idées. Pour Angenot, l'histoire des idées n'est jamais que l'histoire des idées crues et qu'il ne fallait par conséquent aucunement sous-estimer la part de la croyance dans la force des idées. Pour le professeur de McGill, les idées ne vivent que si elles trouvent un terrain d'échange, un espace au cœur duquel elles peuvent se confronter, s'alimenter, évoluer et trouver preneurs chez les fidèles, pour poursuivre la métaphore du sacré employée plutôt par Bouchard. Chez le professeur de Chicoutimi, il en va de même et à cet égard, il nous semble que les deux essais se complètent. Les fameuses idées auxquelles adhère toute une génération ne peut s'expliquer, croyons-nous, que si on comprend les mécanismes sociaux qui alimentent la narration des mythes. Pour Angenot, on ne saura jamais expliquer par le simple biais de la raison comment Hitler put amener avec lui des millions de compatriotes dans son idéologie. La raison qui est hantée par sa volonté de conduire les consciences vers la vérité n'explique pas comment des masses peuvent se mouvoir dans l'erreur. C'est pourquoi Angenot insiste sur le fait que l'on croit souvent en des vérités fausses et c'est cette croyance qui importe dorénavant à expliquer. L'essai de Bouchard va dans la même direction, quand il affirme que le travail de la raison échoue s'il est seul à construire le projet de vérité vers lequel tend le discours. «La construction des identités nationales - à savoir la mobilisation des masses autour des symboles d'appartenance, de mémoire et de solidarité - exigeait un appel aux émotions et au mythe. Comment le travail de la raison, à lui seul, aurait-il pu opérer efficacement sur ces populations peu alphabétisées? Et même là où la scolarisation était plus avancée, qu'il s'agisse de la promotion de la nation ou de la conscientisation des classes ouvrières, comment 
soulever et rallier durablement les foules sinon en s'adressant au cœur plus qu'à l'esprit?» (p. 68). L'approche de Bouchard fonctionne, elle est convaincante aussi il lui paraît utile de proposer une typologie binaire des mythes, à savoir les mythes directeurs dont la matrice structure «la culture d'une société» et les mythes dérivés qui sont subalternes aux premiers. Ainsi les mythes n'ont pas tous la même espérance de vie et apparaissent dans le discours social en fonction des besoins d'un moment plus ou moins long. «De par leur nature, on s'attend que les mythes directeurs jouissent d'un fort consensus, tandis que les mythes dérivés sont souvent le lieu de divisions et controverses (par exemple, les citoyens peuvent adhérer à des visions différentes de la démocratie, de la justice ou de l'égalité)»(p.167). Bouchard propose aussi une dynamique du mythe, comme s'il était lui-même un acteur social porté au regroupement. Il arrive en effet parfois que les mythes s'unissent dans un même but, comme si chacun trouvait en l'autre une forme de résonance à sa propre narration. Bouchard appelle "archémythe» cette fédération des mythes unis derrière une idée maitresse. Il illustre cette trouvaille notamment à partir du modèle social du Canada anglais construit autour d'une idée directive qu'est celle de donner à cette collectivité une identité qui se détache de celle des Etasuniens. Construite sur un idéal qui puise son imaginaire dans les mythes d'un multiculturalisme ouvert, tolérant, non-violent, porté au compromis, pacifiste, prônant l'égalité pour tous. L'expérience anglocanadienne tend toutefois à démontrer que l'archémythe n'est pas aussi permanent qu'on pourrait l'imaginer. Les quelques années du gouvernement conservateur, nous dit Bouchard, pourraient avoir infirmé sa théorie. «On notera que cet archémythe est peut-être en train de se défaire sous l'effet des politiques du gouvernement Harper (militarisation, restauration des symboles monarchiques, coupe dans les politiques sociales, refus de resserrer le contrôle des armes à feu, essor du conservatisme religieux, etc). Mais il faut se garder de conclure hâtivement; il faudra plus de temps pour déterminer si on se trouve devant un écart ou une tendance lourde» (p. 171). À notre avis, l'exemple anglo-canadien n'affaiblit en rien la théorie de Bouchard sur les imaginaires collectifs. Au contraire, son exemple tend à lui donner raison. Au début de son essai, il spécifie que le mythe a un pouvoir sur un territoire donné. Le mythe a donc son lieu anthropologique, hors de ce lieu il n'a pas d'emprise sur l'identité des gens qui l'habitent. Le mythe, comme les gens, habite le lieu. Et si l'archémythe contribuait à l'identité collective, il démontrerait du même coup une nette fragmentation du territoire symbolique canadien. Une cartographie archétypale pourrait ainsi démontrer un Canada désuni non pas seulement par la langue, mais aussi par des valeurs fondamentales auxquelles le Québec serait parfois bien étranger. L'essai de Gérard Bouchard est de multiples points de vue fort stimulant, il pourrait même aider à créer de nouveaux mythes. 